Ciência Florestal, Santa Maria, v. 26, n. 3, p. 875-887, jul.-set., 2016

ISSN 0103-9954

\title{
PERFIL DO FUSTE DE Anadenanthera peregrina DESCRITO PELO MÉTODO DA ALTURA RELATIVA
}

\author{
PROFILE OF Anadenanthera peregrina BOLE DESCRIBED BY THE RELATIVE HEIGHT METHOD
}

\author{
Valdir Carlos Lima de Andrade ${ }^{1}$ Agostinho Lopes de Souza ${ }^{2}$ Marco Antônio Amaro ${ }^{3}$ \\ Walter da Silva Costa ${ }^{4}$ Carlos Pedro Boechat Soares ${ }^{5}$
}

\begin{abstract}
RESUMO
Neste trabalho, o objetivo foi testar o Método da Altura Relativa na cubagem de árvores em pé de Anadenanthera peregrina. Testaram-se, então, quatro diferentes maneiras de se empregar este método, comparando-os com um modelo de taper selecionado dentre vários outros disponíveis na literatura. Foram utilizados dados de 110 árvores-amostra cubadas em pé por meio do Pentaprisma de Weller. Para descrever o perfil do fuste de árvores de Anadenanthera peregrina, o Método da Altura Relativa apresentou expressiva superioridade quando se usaram todas as árvores e, quando se usaram partes destas, obteve-se o mesmo nível de acurácia que o uso de dados reais de cubagem no ajuste de modelos de taper.
\end{abstract}

Palavras-chave: cubagem rigorosa; taper; tronco de cone; Floresta Atlântica.

\begin{abstract}
The objective of this work was to test the Relative Height Method in the scaling of standing trees of Anadenanthera peregrina. Thus, four different approaches of the use of this method were tested and then compared with a taper model selected among those available in the literature. Data from 110 standing tree samples scaled by the Weller Pentaprism were used. To describe the bole profile of Anadenanthera peregrina trees, the Relative Height Method showed an expressive superiority when all the trees were used, and, when part of them was used, the level of accuracy was the same as that of the use of actual scale data in the adjustment of the taper models.
\end{abstract}

Keywords: accurate scaling; taper; cone trunk or frustum; Atlantic Forest.

\section{INTRODUÇÃO}

O conhecimento do volume de madeira é fundamental para gestão de planos de manejo sustentável de florestas nativas, sobretudo, para aprimorar a quantificação de múltiplos produtos e a conversão de madeira em toras para madeira serrada, principalmente onde existem árvores $\mathrm{e}$ espécies de alto valor comercial madeireiro. Esta situação exige o uso de equações de taper, as quais podem contribuir positiva e significativamente na melhoria do nível de confiabilidade da estimação do volume de fuste comercial e, sobretudo, na obtenção de multiprodutos madeireiros e no aumento do

1 Engenheiro Florestal, Dr., Professor Adjunto da Universidade Federal do Tocantins, Rua Badejos, L7, Chácaras 69/72, Zona Rural, CEP 77402-970, Gurupi (TO), Brasil. vclandrade@uft.edu.br

2 Engenheiro Florestal, Dr., Professor Titular do Departamento de Engenharia Florestal, Universidade Federal de Viçosa, Av. Peter Henry Rolfs, s/n, Campus Universitário, CEP 36570-000, Viçosa (MG), Brasil. alsouza@ufv.br

3 Engenheiro Florestal, Dr., Professor Adjunto do Departamento de Ciências Agrárias, Centro de Ciência Agrárias e da Natureza, Universidade Federal do Acre, Rod. BR364, Km 04, Distrito Industrial, CEP 69920-900, Rio Branco (AC), Brasil. marcoantonioamaro@hotmail.com

4 Engenheiro Florestal, Técnico da Isolux Brasil, Rua Diamante, 190 Ap. 304, CEP 35162-057, Ipatinga (MG), Brasil.wsilvac@isoluxcorsan.com

5 Engenheiro Florestal, Dr., Professor Associado do Departamento de Engenharia Florestal, Universidade Federal de Viçosa, Av. Peter Henry Rolfs, s/n, Campus Universitário, CEP 36570-900, Viçosa (MG), Brasil. csoares@ufv.br

Recebido para publicação em 16/08/2012 e aceito em 1/12/2014

Ci. Fl., v. 26, n. 3, jul.-set., 2016 
coeficiente de rendimento de madeira serrada em tora. O termo taper refere-se ao perfil do tronco de árvores e expressa a taxa de decréscimo do diâmetro no sentido base-topo (HUSCH et al., 2003, CAMPOS e LEITE, 2009). Tal característica das árvores varia em função da espécie, idade, tamanho (diâmetro e altura do fuste comercial), ambiente (sítio) e tratamentos silviculturais.

Para plantações florestais no Brasil, muitos estudos sobre equações de taper têm sido realizados com dados de cubagem de árvores abatidas e utilizados na avaliação, prognose e otimização de diferentes produtos madeireiros, dos quais, dentre muitos outros, pode-se citar: Campos e Ribeiro (1982), Figueiredo-Filho et al. (1996), Abreu et al. (2002), Acerbi-Jr. et al. (2002), Assis et al. (2002), Mendonça et al. (2008) e Souza et al. (2008). Para florestas naturais brasileiras, as quais englobam grande variedade de espécies de valor comercial madeireiro, esses estudos ainda são escassos, dos quais, pode-se citar: Chichorro et al. (2003), Leite et al. (2006), Queiroz et al. (2008) e Carvalho et al. (2010).

É importante enfatizar que a cubagem realizada por meio de corte de árvores em florestas nativas é trabalhosa, onerosa, impactante e exige licença emitida pelo órgão ambiental competente (BRASIL, 1998), sendo proibido o abate de árvores em áreas de preservação permanente (BRASIL, 2002) e em unidades de conservação de proteção integral (BRASIL, 2000).

Diante disso, é fundamental dispor de metodologias que permitam descrever o perfil do tronco de árvores a partir da medição de poucos diâmetros, sem precisar abatê-las. Com este intuito, foi desenvolvido o Método da Altura Relativa, cuja metodologia consiste em gerar equações de taper sem o emprego de dados de cubagem rigorosa e abate de árvores-amostra. São utilizados apenas os diâmetros do tronco medidos em $0,3 \mathrm{~m}, 1,3 \mathrm{~m}$, (h-2)/2 e em h (altura total). Essas informações são mensuradas em algumas árvores-amostra em pé, localizadas dentro das parcelas de inventário (ANDRADE et al., 2006).

Diante destas considerações, entende-se que o Método da Altura Relativa pode constituirse de uma metodologia bastante promissora ao uso no cenário de florestas naturais brasileiras, sendo objetivo deste trabalho avaliar este método utilizando dados de Anadenanthera peregrina L. Speg.

\section{MATERIAL E MÉTODOS}

Foram utilizados dados de uma cubagem rigorosa feita em 110 árvores-amostra de Anadenanthera peregrina, coletados na mata da Silvicultura e na mata da Biologia, ambas localizadas no campus da Universidade Federal de Viçosa, em Viçosa - MG. Esta espécie, Anadenanthera peregrina, foi selecionada porque possui ampla ocorrência no Brasil e a madeira tem elevado potencial para produção de multiprodutos, além de tanino, geração de energia, construção civil (interna e externamente) e movelaria, viabilizando a produção sustentável de multiprodutos tanto na pequena e média propriedade rural, bem como a produção em larga escala em diversas regiões do Brasil (LORENZI, 2009; ANDRADE et al., 2013). Além disso, conforme trabalho realizado por Amaro (2010), esta espécie se destaca na área de estudo, apresentando o maior índice de valor de importância e distribuição em várias classes de DAP.

As árvores-amostra de Anadenanthera peregrina foram cubadas em pé pelo método de Smalian, empregando-se o Pentaprisma de Weller a partir de $2 \mathrm{~m}$ de altura e, deste ponto em diante, a cada $2 \mathrm{~m}$ até a base da copa (hf). Nas posições de $0,0 \mathrm{~m}, 1,0 \mathrm{~m}$ e $1,3 \mathrm{~m}$, o diâmetro foi obtido a partir da medição da circunferência com fita métrica e as alturas correspondentes a cada diâmetro foram medidas com vara telescópica de $15 \mathrm{~m}$ de comprimento.

Concluída a coleta de dados, as árvoresamostra foram divididas em dois grupos contendo 55 árvores cada, sendo os dados da Mata da Silvicultura destinado à geração de equações de taper e os dados da Mata da Biologia para aplicação das equações selecionadas. Nesta oportunidade, foram testados cinco métodos para descrever o perfil do fuste de Anadenanthera peregrina, sendo quatro referentes ao Método da Altura Relativa descritos em Andrade (2001) e um referente ao método usual, selecionado dentre os sete modelos de taper apresentados na Tabela 1. Os cinco métodos testados são descritos a seguir:

\section{Método I}

Uso do Método da Altura Relativa aplicando os valores reais dos coeficientes angulares da reta nas equações de taper.

Detalhes dos modelos de taper podem ser vistos em Figueiredo-Filho et al. (1996) e em 
TABELA 1: Modelos de taper avaliados para constituir o método usual.

TABLE 1: Taper models evaluated to make up the usual method.

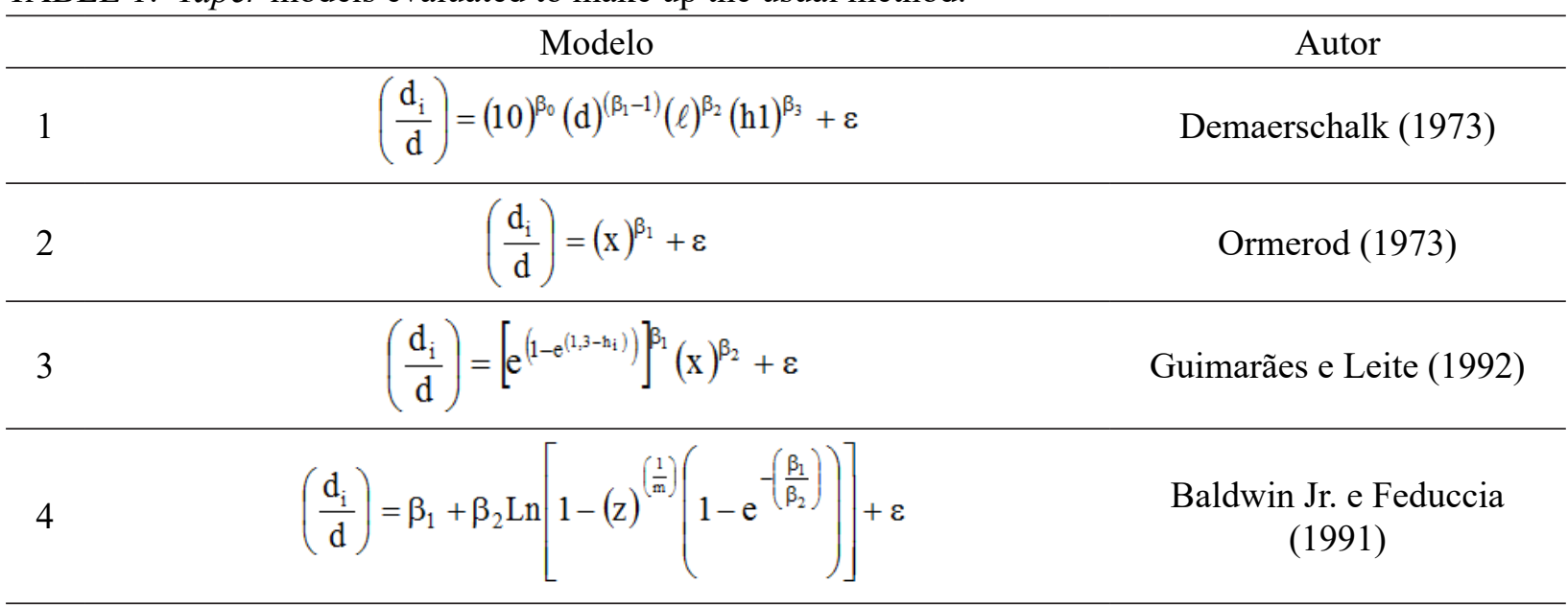

\begin{tabular}{lcc}
$5 \quad\left(\mathrm{~d}_{\mathrm{i}}\right)=\mathrm{a}_{0} \mathrm{~d}^{\mathrm{a}_{1}} \mathrm{a}_{2}^{\mathrm{d}}[(1-\sqrt{\mathrm{z}}) /(1-\sqrt{\mathrm{p}})]^{\left[\beta_{1}(z)^{2}+\beta_{2} \operatorname{Ln}(z+0,001)+\beta_{3} \sqrt{z}+\beta_{4} \mathrm{e}^{(z)}+\beta_{5}\left(\frac{\mathrm{d}}{\mathrm{h}}\right)\right]}+\varepsilon$ & Kozak (1988) \\
\hline $6 \quad\left(\frac{\mathrm{d}_{\mathrm{i}}}{\mathrm{d}}\right)=\left[\beta_{1}(\mathrm{z}-1)+\beta_{2}\left(\mathrm{z}^{2}-1\right)+\beta_{3}\left(\mathrm{a}_{1}-\mathrm{z}\right)^{2} \mathrm{I}_{1}+\beta_{4}\left(\mathrm{a}_{2}-\mathrm{z}\right)^{2} \mathrm{I}_{2}\right]^{0,5}+\varepsilon$ & Max e Burkhart (1976) \\
\hline 7 & $\left(\frac{\mathrm{d}_{\mathrm{i}}}{\mathrm{d}}\right)=\beta_{1}\left[1+\beta_{2} \operatorname{Ln}\left(1-\beta_{3} \mathrm{z}^{\beta_{4}}\right)\right]+\varepsilon$ & Garay (1979)
\end{tabular}

Em que: $\mathrm{d}=$ DAP $(\mathrm{cm}), \mathrm{d}_{\mathrm{i}}=$ diâmetro medido em diferentes partes do tronco com casca $(\mathrm{cm}), \mathrm{h}_{\mathrm{i}}=$ altura na qual se mediu $\mathrm{d}_{\mathrm{i}}(\mathrm{m}), \ell=\mathrm{h} 1-\mathrm{h}_{\mathrm{i}}, \mathrm{z}=\mathrm{h}_{\mathrm{i}} / \mathrm{h}_{1}, \mathrm{x}=\left(\left(\mathrm{h}_{1}-\mathrm{h}_{\mathrm{i}}\right) /\left(\mathrm{h}_{1}-1,3\right)\right), \mathrm{h}_{1}=\mathrm{h}_{\mathrm{f}} / 0,75, \mathrm{Ln}=$ logaritmo neperiano, $\mathrm{a}_{0}, \mathrm{a}_{1}, \mathrm{a}_{2}, \beta_{1} \ldots \beta_{5}=$ parâmetros a serem estimados e $\mathrm{hf}=$ altura até a base da copa, ou, altura de fuste.

Campos e Leite (2009).

\section{Método II}

Uso do Método da Altura Relativa aplicando a análise de regressão para estimar os coeficientes angulares da reta nas equações de taper.

\section{Método III}

Uso do Método da Altura Relativa aplicando a análise de regressão nos diâmetros medidos no tronco para estimar estas variáveis nas equações de taper geradas por este método.

\section{Método IV}

Uso do Método da Altura Relativa para simular dados de cubagem de árvores-amostra em pé, a fim de ajustar o melhor modelo usual de taper.

\section{Método V}

Uso do melhor modelo estatístico usual para gerar equações de taper.

No Método I, é simulada a medição das variáveis em todas as árvores amostradas pelo inventário. Nos Métodos II, III e IV, as variáveis necessárias devem ser medidas em parte das árvores como se faz com a relação hipsométrica em parcelas de inventário. Isto não é possível com o Método $\mathrm{V}$, pois o mesmo exige dados de uma cubagem feita, na maioria dos casos, em árvores-amostra abatidas e em separado das parcelas de inventário.

Para avaliar os cinco métodos de descrição do perfil do fuste de Anadenanthera peregrina, adotou-se a análise da distribuição de resíduos e os seguintes critérios estatísticos:

$$
r\left(y_{i} y_{i}\right)=\left[\frac{\sum_{i=1}^{n} y_{i} y_{i}-\frac{\left(\sum_{i=1}^{n} y_{i}\right)\left(\sum_{i=1}^{n} \hat{y_{i}}\right)}{n}}{\sqrt{\sum_{i=1}^{n}\left(y_{i}-\bar{y}\right)^{2} \sum_{i=1}^{n}\left(y_{i}-\bar{y}\right)^{2}}}\right] 100,
$$




$$
\begin{gathered}
s\left(\mathrm{y}_{\mathrm{i}} \mathrm{y}_{\mathrm{i}}\right)= \pm\left[\frac{\sqrt{\sum_{\mathrm{i}=1}^{\mathrm{n}}\left(\mathrm{y}_{\mathrm{i}}-\hat{y_{i}}\right)^{2} /(\mathrm{n}-2)}}{\overline{\mathrm{y}}}\right] 100 \\
\mathrm{P}_{\%}= \pm \sqrt{\frac{(196)^{2}}{\chi_{\mathrm{n}}^{2}} \sum_{\mathrm{i}=1}^{\mathrm{n}}\left(\frac{\mathrm{y}_{\mathrm{i}}-\hat{y_{i}}}{\mathrm{y}_{\mathrm{i}}}\right)^{2}}
\end{gathered}
$$

Em que: $r\left(y_{i} \widehat{y}_{i}\right)=$ correlação linear, $s\left(y_{i} \widehat{y}_{i}\right)=$ erro padrão residual, $\mathrm{P}_{\%}=$ exatidão determinada pelo teste qui-quadrado $\left(\chi_{n}^{2}\right)$, com nível de significância de $0,05, \mathrm{y}_{\mathrm{i}}$ e $\widehat{y}_{i}=$ diâmetro do tronco real e estimado/ predito, respectivamente, $\mathrm{n}=$ número de pares de $\mathrm{y}_{\mathrm{i}}$ e, $\widehat{y}_{i}$ e $\bar{y}=$ média do diâmetro real do tronco.

Os critérios estatísticos $r\left(y_{i} \widehat{y}_{i}\right), s\left(y_{i} \widehat{y}_{i}\right)$ e $\mathrm{P}_{\%}$ foram englobados em um único percentual (up) aplicando-se:

$$
u p=\frac{\left[100-r\left(y_{i} \hat{y_{i}}\right)\right]+s\left(y_{i} \hat{y_{i}}\right)+P_{\%}}{3}
$$

No desenvolvimento dos Métodos I, II, III e IV, utilizaram-se somente as informações de hf e de diâmetros localizados em $0,0 \mathrm{~m}, 1,3 \mathrm{~m}$, hr e em $\mathrm{hf}$, obtendo-se coeficientes angulares reais da reta aplicando-se as seguintes equações:

$$
\begin{aligned}
& \operatorname{CAR}_{\mathrm{I}}=\frac{\left(1,3-\mathrm{h}_{0,0}\right)}{\left(1-\frac{\mathrm{d}_{\mathrm{h}_{0,0}}}{\mathrm{~d}_{1,3}}\right)} \\
& \mathrm{CAR}_{\mathrm{II}}=\frac{(1,3-\mathrm{hr})}{\left(1-\frac{\mathrm{d}_{\mathrm{hr}}}{\mathrm{d}_{1,3}}\right)} \\
& \mathrm{CAR}_{\mathrm{III}}=\frac{(\mathrm{hr}-\mathrm{hf})}{\left(1-\frac{\mathrm{d}_{\mathrm{hf}}}{\mathrm{d}_{\mathrm{hr}}}\right)} \\
& \mathrm{CAR}_{\mathrm{IV}}=\frac{(1,3-\mathrm{hf})}{\left(1-\frac{\mathrm{d}_{\mathrm{hf}}}{\mathrm{d}_{1,3}}\right)}
\end{aligned}
$$

Em que: $\mathrm{CAR}_{\mathrm{k}}=$ coeficiente angular real da reta que passa pelo $k$-ésimo intervalo I, II, III e IV delimitados em cada uma das 55 árvores-amostra utilizadas para gerar equações de taper, $\mathrm{h}_{0,0}=$ altura na base do tronco referente a $0,0(\mathrm{~m}), \mathrm{d}_{\mathrm{h}_{0,0}}=$ diâmetro medido em $\mathrm{h}_{0,0}(\mathrm{~cm}), \mathrm{hr}=$ altura no tronco obtida por $\mathrm{hr}=$ $(\mathrm{hf}-2) / 2(\mathrm{~m}), \mathrm{d}_{\mathrm{hr}}=$ diâmetro medido em $\mathrm{hr}(\mathrm{cm})$, $\mathrm{d}_{1,3}=$ diâmetro medido em $1,3 \mathrm{~m}(\mathrm{~cm}), \mathrm{hf}=$ altura ou comprimento do fuste $(\mathrm{m})$ e $\mathrm{d}_{\mathrm{hf}}=$ diâmetro medido em hf $(\mathrm{cm})$.

Os coeficientes angulares da reta $\mathrm{CAR}_{\mathrm{k}}$ geram o sólido de revolução cone que, após as devidas transformações algébricas, aplicadas nas equações 1 a 4 , geram as seguintes equações de cones:

$$
\begin{aligned}
& \mathrm{d}_{\mathrm{I}}=\mathrm{d}_{1,3}\left[1-\frac{(1,3-\mathrm{b})}{\mathrm{CAR}_{\mathrm{I}}}\right] \\
& \mathrm{d}_{\text {II }}=\mathrm{d}_{1,3}\left[1-\frac{(1,3-\mathrm{b})}{\mathrm{CAR}_{\mathrm{I}}}\right] \\
& \mathrm{d}_{\text {III }}=\mathrm{d}_{\mathrm{h}}\left[1-\frac{(\mathrm{h}-\mathrm{b})}{\mathrm{CAR}_{\mathrm{III}}}\right] \\
& \hat{\mathrm{d}}_{\mathrm{i}}=\mathrm{d}_{1,3}\left[1-\frac{\left(1,3-\mathrm{h}_{\mathrm{i}}\right)}{\mathrm{CAR}_{\mathrm{IV}}}\right](12)
\end{aligned}
$$

Em que: dc $=$ diâmetro em diferentes partes do cone $(\mathrm{cm}), \mathrm{hc}=$ altura ao longo do cone referente a $d c(\mathrm{~m})$, demais variáveis já foram definidas anteriormente.

As equações de 5 a 8 permitem descrever o perfil do fuste de árvores admitindo-se um nível de acurácia que resulte no mínimo possível de desvios do seu perfil real. Esta condição permite assumir, em média, que as equações de cone 5 a 8 referemse às seguintes equações de taper, respectivamente:

$$
\begin{aligned}
& \hat{d}_{\mathrm{i}}=\mathrm{d}_{1,3}\left[1-\frac{\left(1,3-\mathrm{h}_{\mathrm{i}}\right)}{\mathrm{CAR}_{\mathrm{I}}}\right] \\
& \hat{\mathrm{d}}_{\mathrm{i}}=\mathrm{d}_{1,3}\left[1-\frac{\left(1,3-\mathrm{h}_{\mathrm{i}}\right)}{\mathrm{CAR}_{\mathrm{II}}}\right] \\
& \hat{\mathrm{d}_{\mathrm{i}}}=\mathrm{d}_{\mathrm{hr}}\left[1-\frac{\left(\mathrm{hr}-\mathrm{h}_{\mathrm{i}}\right)}{\mathrm{CAR}_{\mathrm{III}}}\right] \\
& \hat{\mathrm{d}}_{\mathrm{i}}=\mathrm{d}_{1,3}\left[1-\frac{\left(1,3-\mathrm{h}_{\mathrm{i}}\right)}{\mathrm{CAR}_{\mathrm{IV}}}\right]
\end{aligned}
$$

Em que: $\hat{d}_{i}=$ diâmetro de um cone que, em média, pode ser assumido como o diâmetro ao longo do tronco segmentado de árvores, $h_{i}=$ altura referente a $\hat{\mathrm{d}}_{\mathrm{i}}$, demais variáveis já definidas anteriormente.

$\mathrm{Na}$ avaliação das equações 9 a 12 , 
inicialmente, os dados de cubagem das 55 árvoresamostra da mata da Silvicultura foram separados em segmentos do fuste, conforme descridos a seguir:

\section{Porção Basal (PB)}

Segmento do fuste que considera os diâmetros medidos nas posições situadas entre $0,0 \mathrm{~m}$ a $1,3 \mathrm{~m}\left(0,0 \leq \mathrm{h}_{\mathrm{i}} \leq 1,3\right)$.

\section{Porção Mediana (PM)}

Segmento do fuste que considera os diâmetros medidos nas posições situadas entre $1,3 \mathrm{~m} \mathrm{a} \mathrm{hr} \mathrm{m}_{1} \mathrm{~m}\left(1,3<\mathrm{hi} \leq \mathrm{hr}_{1}\right)$.

\section{Porção Superior (PS)}

Segmento do fuste que considera os diâmetros medidos nas posições situadas entre $\mathrm{hr}_{1} \mathrm{ma} \mathrm{hr}_{2} \mathrm{~m}\left(\mathrm{hr}_{1}<\mathrm{h}_{\mathrm{i}} \leq \mathrm{hr}_{2}\right)$.

\section{Porção Apical (PA)}

Segmento do fuste que considera os diâmetros medidos nas posições situadas entre $\mathrm{hr}_{2} \mathrm{~m}$ a hf $\mathrm{m}\left(\mathrm{hr}_{2}<\mathrm{h}_{\mathrm{i}} \leq \mathrm{hf}\right)$.

Aplicando as equações 9 a 12 de forma individual e em suas diferentes combinações, separadamente, em cada segmento do fuste descrito acima, selecionou-se a equação, ou, combinações de equações mais apropriadas para descrever o perfil do fuste de Anadenanthera peregrina. Nesta seleção de equações segmentadas de taper, conforme feito por Andrade (2001), analisou-se a distribuição de resíduos e o Erro Médio Percentual, obtido por:

$$
\operatorname{EMP}=\left(\frac{1}{n}\right) \sum_{\mathrm{i}=1}^{\mathrm{n}}\left(\frac{\hat{\mathrm{d}}_{\mathrm{i}}-\mathrm{d}_{\mathrm{i}}}{\mathrm{d}_{\mathrm{i}}}\right) 100
$$

Em que $\mathrm{d}_{\mathrm{i}}$ é o diâmetro real medido ao longo do fuste das 55 árvores-amostra da Mata da Silvicultura, utilizadas para gerar equações de taper e $\hat{d}_{i}$ já foi definido anteriormente.

Além de as equações de taper 9 a 12 serem estudadas com todas as 55 árvores da Mata da Silvicultura incluídas em um único lote de dados, realizou-se este estudo também utilizando estas mesmas 55 árvores-amostra, separadamente, por classe de altura do fuste dividindo-se os dados em quatro classes, quais sejam: $\mathrm{hf} \leq 9 \mathrm{~m}, 9 \mathrm{~m}<\mathrm{hf} \leq 13 \mathrm{~m}$, $13 \mathrm{~m}<\mathrm{hf} \leq 17 \mathrm{~m}$ ehf $>17 \mathrm{~m}$. Assim, conforme ilustrado na Figura 1, as equações 9 a 12 constituíram duas diferentes situações para serem usadas na descrição do perfil do fuste de Anadenanthera peregrina, sendo:

\section{Situação a}

Utilizou-se o tronco de cone para descrever o fuste segmentado das árvores de Anadenanthera peregrina incluídas em um único lote de dados.

\section{Situação b}

Utilizou-se o tronco de cone para descrever o fuste segmentado das árvores de Anadenanthera peregrina, separadamente, por classe de hf.
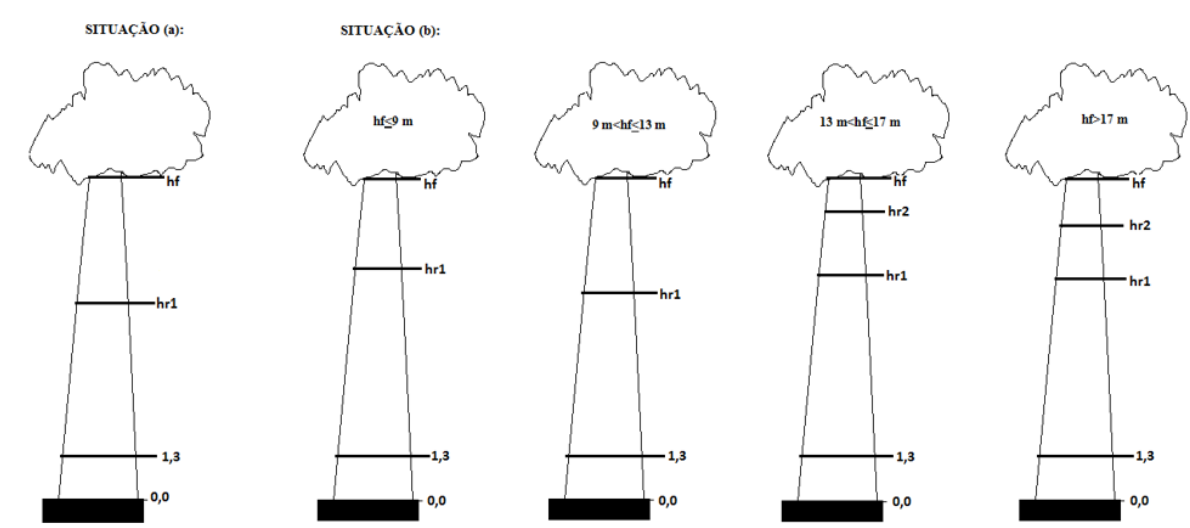

FIGURA 1: Desenho esquemático mostrando as duas situações de uso do Método da Altura Relativa na descrição do perfil do fuste de árvores de Anadenanthera peregrina, em Viçosa - MG.

FIGURE 1: Schematic drawing showing the two situations using of the Relative Height Method to describe the profile of the bole of the trees of Anadenanthera peregrina. 


\section{RESULTADOS E DISCUSSÃO}

A análise das equações 9 a 12, considerandose o menor valor da estatística EMP e a distribuição de resíduos, resultou na avaliação de 17 equações de taper aplicadas em cada uma das quatro porções em que se segmentou o fuste das árvores, separadamente em classes de hf (situação $b$ ) e sem esta separação de classes (situação $a$ ). Nesta análise, seguindo-se o mesmo procedimento relatado por Andrade (2001), obteve-se os resultados apresentados na Tabela 2.

Dentre as duas situações de uso do Método da Altura Relativa, decidiu-se pela situação $a$ como a mais adequada para compor o Método I, pois apresenta o menor valor da estatística up (Tabela $3)$. Nota-se que as estatísticas $r\left(y_{i} \widehat{y}_{i}\right), s\left(y_{i} \widehat{y}_{i}\right)$ e $\mathrm{P}(\%)$, apesar de pouca expressividade, apresenta ram resultados favoráveis ao uso de dados agrupados, sem separar por classe de hf. Este resultado, aliado ao fato de exigir menos tempo e conteúdo de análises que a situação $b$, indica a situação $a$ como a melhor opção para compor o Método I, além disso, ambas as situações $a$ e $b$ apresentaram a mesma tendência na distribuição dos resíduos (Figura 2).

Diante disso, para descrever a perfil do fuste de Anadenanthera peregrina por meio do Método I, deve-se desenvolver o Método da Altura
Relativa, tendo todos os dados agrupados em um único lote e considerando o tronco segmentado em três partes, uma vez que nos segmentos PS e PA decidiu-se utilizar a mesma equação, isto é, a equação 11 (Tabela 2). Assim, os segmentos do fuste considerados, com as suas respectivas equações de taper, são os seguintes:

\section{Porção Basal (PB)}

Refere-se às posições no fuste no intervalo compreendido entre $0,0 \mathrm{~m}$ a $1,3 \mathrm{~m}\left(0,0 \leq \mathrm{h}_{\mathrm{i}}<1,3\right)$ com aplicação da equação 9.

\section{Porção Mediana (PM)}

Refere-se às posições no fuste no intervalo compreendido entre $1,3 \mathrm{~m} \mathrm{a} \mathrm{hr}_{1}=0,55 \mathrm{hf} \mathrm{m}$ $\left(1,3<\mathrm{h}_{\mathrm{i}} \leq 0,55 \mathrm{hf}\right)$ com aplicação da média entre as equações 10 e 11.

\section{Porção Superior e Apical (PSA)}

Refere-se às posições no fuste entre $\mathrm{hr}_{1}=0,55 \mathrm{hf}$ a hf $\left(\mathrm{hr}_{1}<\mathrm{h}_{\mathrm{i}}<\mathrm{hf}\right)$ com aplicação da equação 11 .

Tendo-se decidido pela situação $a$ de

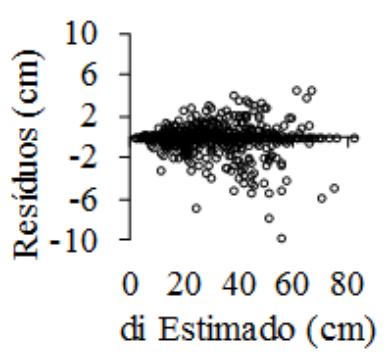

Dados sem separar por classe de hf-situação $a$
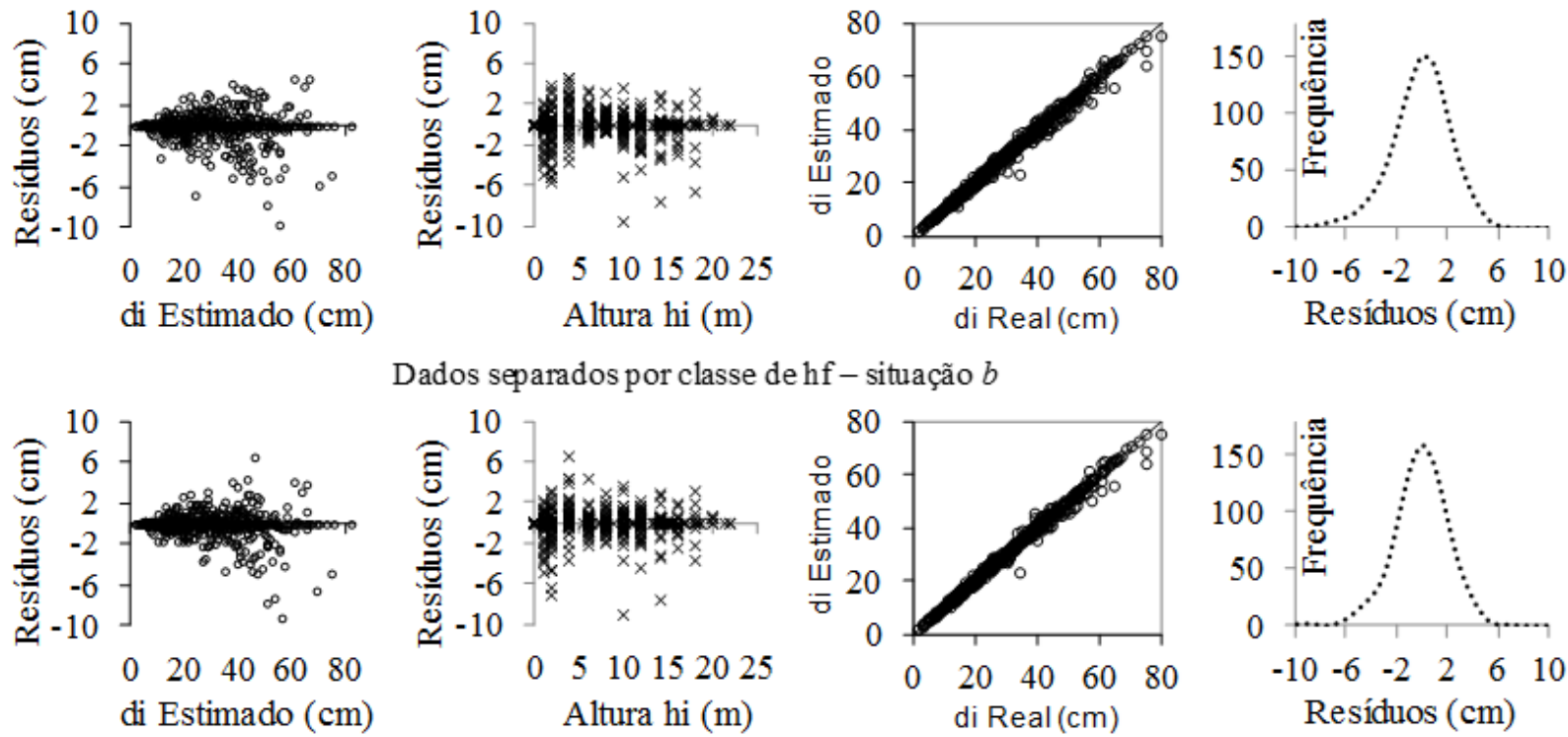

Dados separados por classe de hf - situação $b$

FIGURA 2: Distribuição de resíduos para as situações $a$ e $b$ de emprego do Método da Altura Relativa na descrição do perfil do fuste de árvores de Anadenanthera peregrina na Mata da Silvicultura, em Viçosa - MG.

FIGURE 2: Residual distribution for "a and b" situations of the using the Relative Height Method to drescribe the stem profile of bole trees of Anadenanthera peregrine, Mata da Silvicultua, Viçosa, MG state. 
TABELA 2: Resultados obtidos para as duas situações avaliadas de emprego do Método da Altura Relativa na descrição do perfil do fuste de árvores de Anadenanthera peregrina, em Viçosa - MG.

TABLE 2: Results obtained for the two situations evaluated by the Relative Height Method used in the description of the profile of Anadenanthera peregrina bole trees.

\begin{tabular}{|c|c|c|c|c|c|}
\hline Classe de hf & $\mathrm{PB}\left(0,0 \leq \mathrm{h}_{\mathrm{i}} \leq 1,3\right)$ & \multicolumn{2}{|c|}{$\operatorname{PM}\left(1,3<\mathrm{h}_{\mathrm{i}} \leq \mathrm{hr}_{1}\right)$} & PS $\left(\mathrm{hr}_{1}<\mathrm{h}_{\mathrm{i}} \leq \mathrm{hr}_{2}\right)$ & $\mathrm{PA}\left(\mathrm{hr}_{2}<\mathrm{h}_{\mathrm{i}} \leq \mathrm{hf}\right)$ \\
\hline \multicolumn{6}{|c|}{ Situação (a): } \\
\hline Todas & equação 9 & \multicolumn{2}{|c|}{$\begin{array}{l}\text { Média entre as equações } \\
10 \mathrm{e} 11 ; \mathrm{hr}_{1}=0,55 \mathrm{hf}\end{array}$} & equação 11 & equação 11 \\
\hline \multicolumn{6}{|c|}{ Situação (b): } \\
\hline $\mathrm{hf} \leq 9$ & equação 9 & $\begin{array}{c}\text { equação } \\
11 ; \mathrm{hr}_{1}=0,8 \mathrm{hf}\end{array}$ & $\begin{array}{l}\text { Méd } \\
\text { equaç }\end{array}$ & $\begin{array}{l}\text { entre as } \\
\text { es } 11 \text { e } 12\end{array}$ & $\begin{array}{l}\text { Média entre as } \\
\text { quações } 11 \text { e } 12\end{array}$ \\
\hline $9<\mathrm{hf} \leq 13$ & equação 9 & \multicolumn{2}{|c|}{$\begin{array}{l}\text { Média entre as equações } \\
10,11 \text { e } 12 ; \mathrm{hr}_{1}=0,6 \mathrm{hf}\end{array}$} & Equação 12 & Equação 12 \\
\hline $13<\mathrm{hf} \leq 17$ & equação 9 & \multicolumn{2}{|c|}{$\begin{array}{l}\text { Média entre as equações } \\
10,11 \mathrm{e} 12 ; \mathrm{hr}_{1}=0,8 \mathrm{hf}\end{array}$} & $\begin{array}{c}\text { equação 11; } \\
\mathrm{hr}_{2}=\left(\mathrm{hf}^{\left.-\mathrm{hr}_{1}\right)}\right) 0,7+\mathrm{hr}_{1}\end{array}$ & equação 12 \\
\hline $\mathrm{hf}>17$ & equação 9 & \multicolumn{2}{|c|}{ equação $11 ; \mathrm{hr}_{1}=0,8 \mathrm{hf}$} & $\begin{array}{c}\text { Média entre as } \\
\text { equações } 10 \text { e } 12 ; \\
\mathrm{hr}_{2}=\left(\mathrm{hf}-\mathrm{hr}_{1}\right) 0,45+\mathrm{hr}\end{array}$ & equação 11 \\
\hline
\end{tabular}

TABELA 3: Resultados dos critérios estatísticos adotados para avaliar as situações $a$ e $b$ de emprego do Método da Altura Relativa na descrição do perfil do fuste de árvores de Anadenanthera peregrina na Mata da Silvicultura, em Viçosa - MG.

TABLE 3: Results of the statistic criteria used to determine $a$ and $b$ employment situations the Relative Height Method in description of the profile of bole of the trees Anadenanthera peregrina in Mata da Silvicultura in Viçosa, MG state.

\begin{tabular}{lccccc}
\hline \multicolumn{1}{c}{ Situações } & EMP & $r\left(y_{i} \widehat{y}_{i}\right)$ & $s\left(y_{i} \widehat{y}_{i}\right)$ & $\mathrm{P}(\%)$ & up \\
\hline dados sem separar por classe de hf $($ situação $a)$ & $-0,079$ & 99,17 & 4,87 & 7,73 & 4,48 \\
dados separados por classe de hf $($ situação $b$ ) & $-0,211$ & 99,04 & 5,24 & 8,45 & 4,88 \\
\hline
\end{tabular}
Em que: EMP = erro médio percentual, up = único percentual, $r\left(y_{i} \widehat{y}_{i}\right)=$ correlação linear, $s\left(y_{i} \widehat{y}_{i}\right)=$ erro padrão residual, $\mathrm{P}_{\%}=$ exatidão determinada pelo teste qui-quadrado $\left(\chi_{n}^{2}\right)$ com nível de significância de $0,05, \mathrm{y}_{\mathrm{i}}$ e $\widehat{y}_{i}=$ diâmetro do tronco real e estimado/predito, respectivamente.

desenvolvimento do Método da Altura Relativa (Método I), procedeu-se ao ajuste e análise dos sete modelos de taper apresentados na Tabela 1. Assim, empregando-se os mesmos dados das 55 árvoresamostra da Mata da Silvicultura, obtiveram-se os resultados apresentados na Tabela 4 que indicam o Modelo 5, modelo de Kozak (1988), como o mais adequado para constituir o Método V (menor valor da estatística up).

Para o Modelo 4, selecionou-se $\mathrm{m}=3$ e, para o Modelo 5, $\mathrm{p}=0,25$. Em que: $\mathrm{a}_{0}, \mathrm{a}_{1}, \mathrm{a}_{2}, \beta_{1} \ldots$ $\beta_{5=}$ parâmetros estimados, $r\left(y_{i} \widehat{y}_{i}\right)=$ correlação linear, $s\left(y_{i} \widehat{y}_{i}\right)=$ erro padrão residual, $\mathrm{P}_{\%}=$ exatidão determinada pelo teste qui-quadrado $\left(\chi_{n}^{2}\right) \operatorname{com}$ nível de significância de $0,05, y_{i}$ e $\widehat{y}_{i}=$ diâmetro do tronco real e estimado/predito, respectivamente, up = único percentual.

O Modelo 5 foi, também, utilizado para constituir os Métodos III e IV, sendo no Método III utilizado o modelo linear clássico para estimar o diâmetro $\quad \mathrm{d}_{\mathrm{h}_{0,0}}$ e o Modelo 5 para estimar os diâmetros $d_{\text {ln }}$ e $d_{\text {lf }}$. Já para compor o Método II, empregou-se a análise de regressão de $\mathrm{CAR}_{\mathrm{k}}=\mathrm{f}(\mathrm{d} ; \boldsymbol{h})$. As equações de taper geradas para os cinco métodos avaliados são as seguintes: 
TABELA4: Parâmetros estimados e estatísticas utilizados para avaliar sete modelos de taper para descrever o perfil do fuste de Anadenanthera peregrina, Mata da Silvicultura, Viçosa - MG.

TABLE 4: Estimated parameters and statistics used to evaluated seven taper models to describe profile of the bole trees of Anadenanthera peregrina, Mata da Silvicultura, Viçosa-MG state.

\begin{tabular}{|c|c|c|c|c|c|c|c|}
\hline \multicolumn{8}{|c|}{ Modelo } \\
\hline & 1 & 2 & 3 & 4 & 5 & 6 & 7 \\
\hline$\hat{\mathrm{a}}_{0}$ & & & & & 1,0913 & & \\
\hline$\hat{\mathrm{a}}_{1}$ & & & & & 0,8895 & 0,6184 & \\
\hline$\hat{a}_{2}$ & & & & & 1,0040 & 1,0000 & \\
\hline$\hat{\beta}_{0}$ & 0,0779 & & & & & & \\
\hline$\hat{\beta}_{1}$ & 1,0492 & 0,4181 & $-0,0471$ & 1,1113 & $-0,9999$ & $-9,3976$ & 1,1428 \\
\hline$\hat{\beta}_{2}$ & 0,4173 & & 0,3516 & 0,2223 & 0,0707 & 2,8511 & 4,3398 \\
\hline$\hat{\beta}_{3}$ & $-0,5807$ & & & & $-1,3730$ & 5,7427 & 0,1122 \\
\hline$\hat{\beta}_{4}$ & & & & & 0,9974 & $-7,6375$ & 0,5241 \\
\hline$\hat{\beta}_{5}$ & & & & & $-0,0611$ & & \\
\hline$r\left(y_{i} y_{i}\right)$ & 95,14 & 94,59 & 95,89 & 95,53 & 96,91 & 96,05 & 96,16 \\
\hline$s\left(y_{i} \hat{y_{i}}\right)$ & 11,78 & 12,44 & 10,84 & 11,30 & 9,40 & 10,62 & 10,48 \\
\hline$P_{\%}$ & 24,94 & 25,63 & 24,88 & 24,76 & 22,92 & 25,02 & 25,24 \\
\hline up & 13,86 & 14,49 & 13,28 & 13,51 & 11,80 & 13,20 & 13,19 \\
\hline
\end{tabular}

\section{Método I}

Constitui-se das equações de taper 9, 10 e 11 empregando valores reais de $\mathrm{CAR}_{\mathrm{I}}, \mathrm{CAR}_{\mathrm{II}}$ e $\mathrm{CAR}_{\mathrm{III}}$ obtidos por meio das equações 1,2 e 3, respectivamente, utilizando $d_{\mathrm{h}_{0,0}}, \mathrm{~d}_{\text {ll }}$ e $\mathrm{d}_{\text {lf }}$ medidos em todas as árvores-amostra.

\section{Método II}

Constitui-se das equações de taper 9, 10 e 11 empregando-se valores estimados de $\mathrm{CAR}_{\mathrm{I}}$, $\mathrm{CAR}_{\mathrm{II}}$ e $\mathrm{CAR}_{\mathrm{III}}$, respectivamente, obtidos por meio das seguintes equações selecionadas dentre várias outras:
$\hat{\operatorname{CAR}}_{1}=\mathrm{e}^{0,9859 \mathrm{~d}^{0,3044(\mathrm{E}-1)}}$

$\hat{\mathrm{CAR}}_{2}=2,2-0,00066 \mathrm{~d}(0,5 \mathrm{~K}-1)-5,7516\left(\frac{1}{\mathrm{~d}}\right)+0,5724 \mathrm{~h}$ (f $)$

$\hat{\mathrm{CAR}}_{3}=1-4,7029 \mathrm{e}^{0,000039 \mathrm{~d}(\mathbf{B}, 7096 \mathrm{~h}+3,3241)}$.

\section{Método III}

Constitui-se das equações de taper 9, 10 e 11 empregando valores estimados de $\mathrm{CAR}_{\mathrm{I}}, \mathrm{CAR}_{\mathrm{II}}$ e $\mathrm{CAR}_{\mathrm{III}}$ obtidos por meio das equações 1,2 e 3 , respectivamente, utilizando $\mathrm{d}_{\mathrm{h}_{0,0}}, \mathrm{~d}_{\mathrm{ll}}$ e $\mathrm{d}_{\mathrm{ff}}$ obtidos por meio das seguintes equações: 


$$
\begin{aligned}
& \mathrm{d} b=2,7487+1,1655 \mathrm{~d}-0,2678 \mathrm{hf} ; \\
& \hat{\mathrm{dr}}=0,7991 \mathrm{~d}^{1,0618} 0,9981^{\mathrm{d}}\left(\frac{1-\sqrt{\mathrm{z}}}{1-\sqrt{0,25}}\right)^{-\left[77,4081(z)^{2}+20,5190 \operatorname{Ln}(z+0,001)-113,9 \sqrt{z}+70,1652 \mathrm{e}^{(z)}-0,0887\left(\frac{\mathrm{d}}{\mathrm{h} 1}\right)\right]} \\
& \mathrm{Com} \mathrm{z}=\frac{\mathrm{hr}}{\mathrm{h} 1} ; \mathrm{e} \\
& \hat{\mathrm{df}}=0,7991 \mathrm{~d}^{1,0618} 0,9981^{\mathrm{d}}\left(\frac{1-\sqrt{\mathrm{z}}}{1-\sqrt{0,25}}\right){ }^{\left.-77,4081(z)^{2}+20,5190 \operatorname{Ln}(z+0,001)-113,9 \sqrt{z}+70,1652 \mathrm{e}^{(z)}-0,0887\left(\frac{\mathrm{d}}{\mathrm{h} 1}\right)\right]} \\
& \mathrm{Com} \mathrm{z}=\frac{\mathrm{hf}}{\mathrm{h} 1}
\end{aligned}
$$

\section{Método IV}

Constitui-se da seguinte equação de taper, gerada com dados de cubagem simulados pelo Método da Altura Relativa (situação $a$ ), com z $=\mathrm{h}_{\mathrm{i}} / \mathrm{h}_{1}$ :

\section{Método V}

$$
\hat{d_{i}}=0,9681 d^{0,9366} 1,0024 d\left(\frac{1-\sqrt{z}}{1-\sqrt{0, Z}}\right)^{\left[-0,9367(z)^{2}+0,0481 \text { h }(z+0,001)-1,1272 \sqrt{z}+0,8566 \mathrm{e}^{(z)}-0,0499\left(\frac{d}{h 1}\right)\right]}
$$

Constitui-se da seguinte equação de taper, gerada com dados reais de cubagem, com $\mathrm{z}=\mathrm{h}_{\mathrm{i}} / \mathrm{h}_{1}$ :

$$
\hat{d_{i}}=1,0913 d^{0,8895} 1,0040^{d}\left(\frac{1-\sqrt{z}}{1-\sqrt{0, Z}}\right)^{\left[-0,9999(z)^{2}+0,0707 \mathrm{~h}(z+0,001)-1,3730 \sqrt{z}+0,9974 \mathrm{e}^{(z)}-0,0611\left(\frac{d}{\mathrm{~h} 1}\right)\right]} .
$$

Os resultados dos critérios estatísticos de análise, obtidos para os Métodos II, III e IV, são apresentados na Tabela 5. Já, para os Métodos I e V, tais resultados foram apresentados nas Tabelas $3 \mathrm{e}$ 4 , respectivamente. Nota-se que na comparação do Método V com o Método I, houve uma expressiva superioridade deste último com up de 4,48\% (Tabela 3) contra $11,80 \%$ (Tabela 4).

Por outro lado, ao simular o uso do Método da Altura Relativa similar ao que se faz com a relação hipsométrica em parcelas de inventário, no qual se mede a altura de apenas parte das árvores, o Método III (Tabela 5) resultou na melhor opção de emprego do método da altura, pois teve menor valor da estatística up, isto é, $10,49 \%$ contra $13,40 \%$ e $11,93 \%$, respectivamente, Método III contra Métodos II e IV. Neste caso, dentre os Métodos II, III e IV, o Método III, que se refere ao emprego do Método da Altura Relativa utilizando a análise de regressão nos diâmetros $\mathrm{d}_{\mathrm{h}_{0,0}}, \mathrm{~d}_{\mathrm{ln}}$ e $\mathrm{d}_{\text {li }}$, é o mais adequado para descrever o perfil do fuste de Anadenanthera peregrina na Mata da Silvicultura, em Viçosa - MG.

O ajuste do Modelo 5 (KOZAK, 1988) utilizando dados de cubagem simulados pelo Método da Altura Relativa (Método IV), além de pouca diferença nas estatísticas de $r\left(y_{i} \widehat{y}_{i}\right), \quad s\left(y_{i} \widehat{y}_{i}\right)$, $\mathrm{P}_{\%}$ e, consequentemente, up, teve a mesma tendência na distribuição de resíduos (Figura 3) que o ajuste utilizando dados reais de cubagem (Método V). Esta similaridade de resultados estatísticos e de dispersão de resíduos, também, foi evidenciada por Andrade (2001) com o modelo de Demaerschalk (1973). Portanto, não é necessário abater árvores-amostra para cubagem, ou fazê-la com árvores-amostra em pé. Neste caso, pode-se usar o Método da Altura Relativa por meio do Método IV, cujas medições exigidas podem ser feitas com a árvore em pé nas posições: $0,0 \mathrm{~m}$ e $(\mathbf{h}-2) / 2$, além de $1,3 \mathrm{~m}$ e de hf. 
TABELA 5: Resultados dos critérios estatísticos para os Métodos II, III e IV utilizando os mesmos dados das 55 árvores-amostra de Anadenanthera peregrina da Mata da Silvicultura, em Viçosa MG, empregados para gerar equações de taper.

TABLE 5: Results of the statistic criteria for the methods II, III and IV using the same data from 55 sample trees of Anadenanthera peregrina used to produce the taper equations.

\begin{tabular}{cccc}
\hline & Método II & Método III & Método IV \\
\hline $\mathrm{r}\left(\mathrm{y}_{\mathrm{i}} \mathrm{y}_{\mathrm{i}}\right)$ & 96,56 & 97,27 & 96,92 \\
$\mathrm{~s}\left(\mathrm{y}_{\mathrm{i}} \mathrm{y}_{\mathrm{i}}\right)$ & 9,91 & 8,84 & 9,38 \\
\hline$P_{\%}$ & 26,85 & 19,92 & 23,34 \\
up & 13,40 & 10,49 & 11,93 \\
\hline
\end{tabular}

Em que: $r\left(y_{i} y_{i}\right)=$ correlação linear, $s\left(y_{i} y_{i}\right)=$ erro padrão residual, $P_{\%}=$ exatidão determinada pelo teste qui-quadrado $\left(\chi_{n}^{2}\right)$ com nível de significância de $0,05, \mathrm{y}_{\mathrm{i}}$ e $\hat{\mathrm{y}_{\mathrm{i}}}=$ diâmetro do tronco real e estimado/predito, respectivamente, up $=$ único percentual.

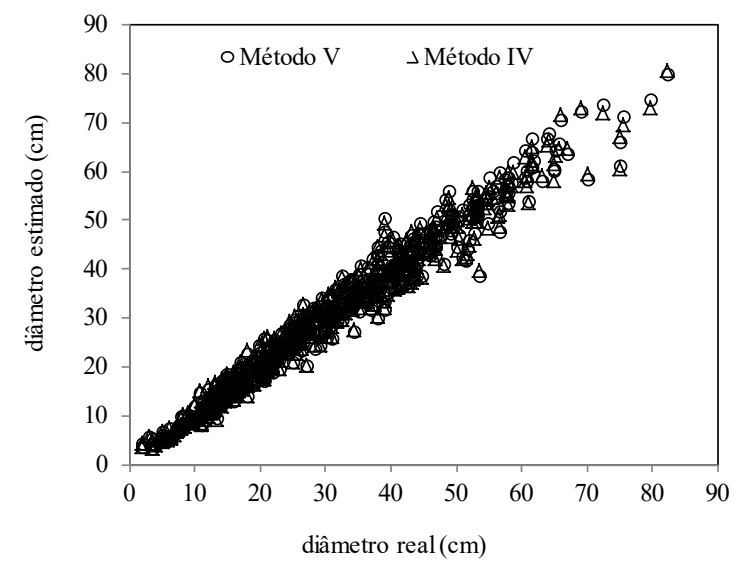

FIGURA 3: Dispersão de diâmetros do fuste de Anadenanthera peregrina empregando o Modelo 5 ajustado com dados reais de cubagem (Método V) e dados de cubagem simulados pelo Método da Altura Relativa (Método IV).

FIGURE 3: Diameter dispersion of the Anadenanthera peregrina boles, employing Model 5 adjusted with scaling actual data (V Method) and scaling data simulated by the Relative Height Method (IV Method).

Visando verificar o comportamento dos Métodos I, III, IV e V na predição do perfil do fuste de Anadenanthera peregrina, procedeu-se à aplicação das equações geradas aos dados de 55 árvoresamostra cubadas na Mata da Biologia, cujos dados são independentes, isto é, não foram utilizados para gerar as equações de taper que se constituem nestes métodos. Os resultados obtidos são apresentados na Figura 4 e Tabela 6, nas quais se nota que o Método I foi, expressivamente, melhor que os demais. $\mathrm{O}$ Método IV, desta vez, foi melhor que os Métodos III e V quanto à distribuição de resíduos (Figura 4) e critérios estatísticos (Tabela 6).

\section{CONCLUSÕES}

Pode-se concluir que:

1) Não é necessário desenvolver o Método da Altura Relativa por classe de altura de fuste;

2) Para descrever o perfil do fuste de árvores de Anadenanthera peregrina empregando-se o Método da Altura Relativa, foi suficiente dividilo em três segmentos, quais sejam: porção basal $\left(0,0 \leq \mathrm{h}_{\mathrm{i}}<1,3\right)$, porção mediana $\left(1,3<\mathrm{h}_{\mathrm{i}} \leq 0,55 \mathrm{hf}\right)$, e porção superior $\left(0,55 \mathrm{hf} \leq \mathbf{h}_{\mathrm{i}}<\mathrm{hf}\right)$;

3) Dentre os sete modelos de taper avaliados, o modelo de Kozak (1988) foi o melhor;

4) O ajuste de modelos de taper, empregando-se dados de cubagem simulados pelo Método da Altura Relativa, resulta no mesmo nível de acurácia que o uso de dados reais de cubagem;

5) Quando forem medidos $d_{h_{0,0}}, d_{h}$ e $d_{h}$ em parte de árvores das parcelas de inventário, deve-se preferir o Método III para descrever o perfil do fuste de árvores de Anadenanthera peregrina, ao 
TABELA 6: Resultados dos critérios estatísticos adotados para avaliar os Métodos I, III, IV e V no teste de aplicação com 55 árvores-amostra de Anadenanthera peregrina procedentes da Mata da Biologia localizada em Viçosa - MG, cujos dados são diferentes das 55 árvores-amostra da mesma espécie procedentes da Mata da Silvicultura, Viçosa - MG, utilizadas para gerar equações de taper.

TABLE 6: Results of the statistic criteria for the methods used to evaluated the Methods I, III, IV and IV in the application test with 55 sample trees of Anadenanthera peregrina coming from Mata da Biologia located in Viçosa-MG state, whose data are different from the 55 sample trees of the same species coming from the Mata da Silvicultura used to generate taper equations.

\begin{tabular}{lccccc}
\multicolumn{1}{c}{ Método } & EMP & $\mathrm{r}\left(\mathrm{y}_{\mathrm{i}} \mathrm{y}_{\mathrm{i}}\right)$ & $\mathrm{s}\left(\mathrm{y}_{\mathrm{i}} \hat{\mathrm{y}}_{\mathrm{i}}\right)$ & $\mathrm{P}_{\%}$ & up \\
\hline Método I & 0,242 & 99,25 & 4,46 & 7,33 & 4,18 \\
Método III & 0,512 & 92,82 & 13,76 & 24,52 & 15,16 \\
Método IV & $-0,944$ & 94,71 & 11,81 & 22,47 & 13,19 \\
Método V & $-0,870$ & 93,86 & 12,73 & 23,65 & 14,17 \\
\hline
\end{tabular}

Em que: $\mathrm{EMP}=$ erro médio percentual, $\mathrm{r}\left(\mathrm{y}_{\mathrm{i}} \mathrm{y}_{\mathrm{i}}\right)=$ correlação linear, $\mathrm{s}\left(\mathrm{y}_{\mathrm{i}} \mathrm{y}_{\mathrm{i}}\right)=$ erro padrão residual, $\mathrm{P}_{\%}=$ exatidão determinada pelo teste qui-quadrado $\left(\chi_{n}^{2}\right)$ com nível de significância de $0,05, \mathrm{y}_{\mathrm{i}}$ e $\hat{\mathrm{y}}_{\mathrm{i}}=$ diâmetro do tronco real e estimado/predito, respectivamente, up $=$ único percentual.
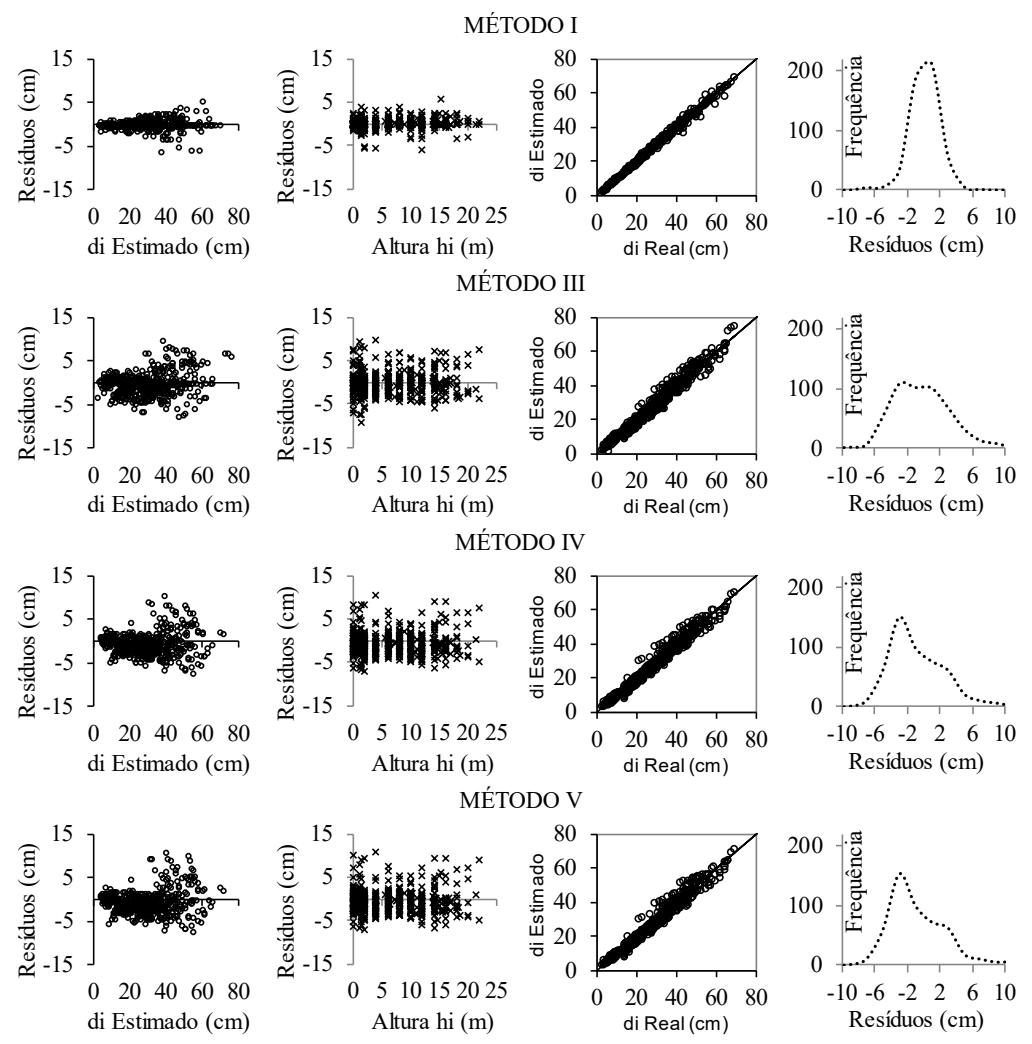

FIGURA 4: Distribuição de resíduos para o perfil do fuste de árvores de Anadenanthera peregrina empregando os Métodos I, III, IV e V em um teste de aplicação com 55 árvores-amostra procedentes da Mata da Biologia, diferentes das 55 árvores-amostra procedentes da Mata da Silvicultura, utilizadas para gerar equações de taper.

FIGURE 4: Residual distribution for the profile of the bole of the trees Anadenanthera peregrina emplying Methods I, III, IV and V in an application test with 55 sample trees coming from Mata da Biologia, different of the 55 sample trees coming from Mata da Silvicultura, used to generate taper equations. 
invés do Método II;

6) Medir informações necessárias ao desenvolvimento do Método da Altura Relativa em todas as árvores resulta em expressiva superioridade, que medir apenas em algumas árvores-amostra.

\section{REFERÊNCIAS BIBLIOGRÁFICAS}

ABREU, E.C.R. et al. Modelagem para prognose precoce do volume por classe diamétrica para Eucalyptus grandis. Scientia Forestalis, n.61, p.86-102, 2002.

ACERBI-JR, F.W. et al. Modelo para prognose do crescimento $\mathrm{e}$ da produção $\mathrm{e}$ análise econômica de regimes de manejo para Pinus taeda L. Revista Árvore, v.26, n.6, p.699-713, 2002.

AMARO, M. A. Estimativas do estoque de volume, biomassa e carbono para fustes de árvores, sub-bosques e serapilheira em uma Floresta Estacional Semidecidual Montana em Viçosa, MG. 2010. 180f. Tese (Doutorado em Ciência Florestal) - Universidade Federal de Viçosa, Viçosa, MG, 2010.

ANDRADE, V.C.L.; CALEGÁRIO, N.; SCOLFORO, J.R.S. Análise de algumas alternativas para obter o coeficiente angular da reta no método da altura relativa. Ciência Florestal, v.16, n.3, p.303-317, 2006.

ANDRADE, B. G. et al. Determinação do potencial tanífero em povoamentos de angico. Ciência da Madeira (Braz. J. Wood Sci.), Pelotas/RS, v.04, n.02, novembro de 2013.

ANDRADE, V. C. L. Um método para descrever o perfil do tronco em árvores de eucalipto utilizando geometria analítica. 2001. 74p. Dissertação (Mestrado em Ciência Florestal) - Universidade Federal de Viçosa, Viçosa, 2001.

ASSIS, A.L. et al. Avaliação de modelos polinomiais segmentados e não-segmentados na estimativa de diâmetro e volumes comerciais de Pinus taeda. Ciência Florestal, v.12, p.89-107, 2002.

BRASIL. Lei no 9.605, de 12 de fevereiro de 1998. Dispõe sobre as sansões penais e administrativas derivadas de condutas e atividades lesivas ao meio ambiente, e dá outras providências. Diário Oficial da União, Brasília, 12 de fevereiro de 1998.

BRASIL. Lei № 9.985, de 18 de julho de 2000 .
Institui o Sistema Nacional de Unidades de Conservação da Natureza (SNUC). Estabelece critérios e normas para a criação, implantação e gestão das unidades de conservação. Diário Oficial da União, Brasília; 19 de julho de 2000.

BRASIL. CONAMA - CONSELHO NACIONAL DO MEIO AMBIENTE. Resolução $\mathrm{N}^{\circ}$ 303, de 20 de março de 2002. Dispõe sobre parâmetros, definições e limites de Áreas de Preservação Permanente. Diário Oficial da União, Brasília; 13 de maio de 2002.

CAMPOS, J.C.C; LEITE, H.G. Mensuração florestal: perguntas e respostas. Viçosa: UFV, 3ed., 2009. 548p.

CAMPOS, J.C.C., RIBEIRO, J. C. Avaliação de Dois Modelos de "taper" em Árvores de Pinus patula. Revista Árvore, Viçosa, v.6, n.2, p.140-149, 1982.

CARVALHO, S. P. C. et al. Diferentes estratégias para estimar o volume comercial de Anadenanthera colubrina (Vell.) Brenan. Cerne, v.16, n.3, p.399406. 2010.

CHICHORRO, J.F.; RESENDE, J.L.P.; LEITE, H.G. Equações de volume e de taper para quantificar multiprodutos da madeira em Floresta Atlântica. Revista Árvore, v.27, n.6, p.799-809, 2003.

FIGUEIREDO-FILHO, A.; BORDERS, B.E.; HITCH, K.L. Taper equations for Pinus taeda plantations in Southern Brazil. Forest Ecology and Management, v.83, p.39-46, 1996.

HUSCH, B.; BEERS, T. W.; KERSAW JR., J. A. Forest mensuration. $4^{\text {th }}$ ed. New Jersey: John Wiley \& Sons, 443p. 2003.

KOZAK, A. A variable-exponent taper equation. Canadian Journal of Forest Research. v.18, p.1363-1368, 1988.

LEITE, H. G. et al. Função de afilamento para Virola surinamensis (Roll.) Warb. Revista Árvore, v.30, n.1, p.99-106, 2006.

LORENZI, H. Árvores Brasileiras: manual de identificação e cultivo de plantas arbóreas nativas do Brasil. 3.ed. Nova Odessa: Instituto Plantarum, 2009. v. 2, 384 p.

MENDONÇA, A.R. et al. Avaliação de um sistema para otimização do sortimento de Eucalyptus sp. Ciência Florestal, v.18, n.2, p. 247-258, 2008.

QUEIROZ, D. et al. Identidade de modelos em funções de afilamento para Mimosa Scabrella 
Bentham em povoamentos nativos da região SOUZA, C. A. M. et al. Modelos de afilamento para metropolitana de Curitiba/PR. Floresta, v.38, n.2, o sortimento do fuste de Pinus taeda L. Ciência P.339-349, 2008.

Rural, v.38, n.9, dez, p.2506-2511, 2008. 